\title{
Up to fifth-order Raman scattering of InP under nonresonant conditions
}

\author{
L. Artús and R. Cuscó \\ Institut Jaume Almera, Consell Superior d'Investigacions Científiques (CSIC), Martí i Franquès s.n., 08028 Barcelona, Spain \\ J. M. Martín, and G. González-Díaz \\ Departamento de Electricidad y Electrónica, Facultad de Física, Universidad Complutense, 28040 Madrid, Spain
}

(Received 15 March 1994)

\begin{abstract}
We present Raman spectra of InP measured under nonresonant conditions revealing multiphonon processes up to fifth order. Using an incident photon energy in the absorption region of the compound but far from any of its interband transitions, nonresonant multiphonon processes of order higher than two, which have not been reported so far in a zinc-blende-type semiconductor, have been observed in indium phosphide. In this way it has been possible to detect contributions not only from the longitudinal optical phonons but also from the transverse optical phonons in the higher-order peaks. We find a very good agreement between multiples of the TO- and LO-phonon frequencies at the zone center and the higher-order phonons measured in the experiments. The trend of strong intensity reductions observed when passing from first to second as well as from second to third order is not maintained when going from third to fourth, and from fourth to fifth order.
\end{abstract}

\section{INTRODUCTION}

To date, multiphonon processes in semiconductors have been observed in Raman scattering experiments only under resonant conditions. Thus, several studies on bulk wurzite and zinc-blende II-VI compounds deal with multiphonon processes measured by means of resonant Raman scattering. Overtones up to order $n=4-9$ have been reported for $\mathrm{CdS},{ }^{1} \mathrm{ZnSe}$, and $\mathrm{ZnTe},{ }^{2} \mathrm{ZnS},{ }^{3}$ and CdTe. ${ }^{4}$ By contrast, only a few multiphonon studies are available on III-V compounds, notably on GaP, for which up to third-order resonant Raman scattering has been observed. ${ }^{2}$ Magneto-Raman experiments have shown that the resonant multiphonon lines are strongly enhanced in suitable magnetic fields, making it possible to observe up to seven LO phonons in GaAs (Ref. 5) and four LO phonons in InP. ${ }^{6}$ However, under resonant conditions only $n$ LO phonons can be detected, and therefore multiphonon processes with contribution of transverse phonons cannot be observed using these techniques.

To our knowledge, no experimental evidence of nonresonant Raman scattering for orders higher than two has been reported on III-V and II-VI semiconductors so far. The aim of this work is to prove that the observation of high-order Raman scattering is possible under nonresonant conditions taking advantage of the high sensitivity of photon detectors currently available. Indium phosphide was chosen for this study because of the flatness not only of its transverse-optical branch but also of its longitudinal-optical branch, which gives rise to a high density of states at frequencies close to those of zonecenter TO and LO phonons. ${ }^{7}$ Beside the resonant Raman-scattering experiments in magnetic field mentioned above, complete ${ }^{8,9}$ and partial ${ }^{10}$ second-order Raman spectra of InP out of resonance have also been published. Raman measurements performed on InP above the band gap enabled us to detect up to fifth-order Raman scattering showing the presence of transverse-optical as well as longitudinal-optical phonons in the higherorder spectra.

\section{EXPERIMENT}

The experiments were performed at room temperature on a (100) face of liquid-encapsulated Czochralski grown, semi-insulating (Fe-doped to approximately $5 \times 10^{16}$ $\mathrm{cm}^{-3}$ ) InP samples supplied by Sumitomo. For such a low doping level the Raman scattering by intrinsic InP modes is not altered by the presence of the impurities. Prior to the experiments, the surface was etched in a $\mathrm{H}_{2} \mathrm{O}_{2}: \mathrm{H}_{3} \mathrm{PO}_{4}$ solution for $1 \mathrm{~min}$, rinsed with de-ionized water and methanol, and blown dry with argon. The Raman measurements were performed using a T64000 Jobin-Yvon spectrometer equipped with a chargecoupled device detector cooled with liquid nitrogen. The incident line we used was the 4880 - $\AA$ line of an argon-ion laser, whose photon energy lies within the absorption region of the material but far from its different interband transitions. The incident power on the sample was about $220 \mathrm{~mW}$. The spectra were recorded using the double subtractive configuration of the spectrometer, with 200$\mu \mathrm{m}$ slits. For the fourth and fifth orders, the sample area was purged with a continuous argon flow in order to suppress the Raman-scattering lines arising from interactions with atmospheric $\mathrm{O}_{2}$ in the region around 1500 $\mathrm{cm}^{-1}$. The integration time for a single measurement was $40 \mathrm{~min}$. The third-order spectrum was obtained by adding 13 single measurements, whereas in the fourthand fifth-order spectra 17 of those measurements were added.

\section{RESULTS}

In order to determine the TO and LO frequencies with higher accuracy, the first-order Raman spectrum was recorded using the triple additive configuration of the 
spectrometer with a resolution of $\approx 1 \mathrm{~cm}^{-1}$. In this way, a more accurate comparison between the multiples of these phonons and the measured higher-order spectra could be established. Typical first-order TO and LO frequencies were found to be 306.9 and $344.6 \mathrm{~cm}^{-1}$, respectively. The TO mode is very weak in relation to the LO mode since it is forbidden in backscattering configuration on a (100) face.

Figure 1 shows second- and third-order Raman spectra of InP. As it can be noted, the different Raman spectra displayed in this paper were recorded without polarization analysis. In studies of second-order Raman scattering, different polarization configurations are usually considered in order to separate the various irreducible components of the two-phonon spectrum. In zinc-blendetype semiconductors it is generally found that, when the $\Gamma_{1}$ component is measured, overtones dominate the second-order spectrum whereas combinations are better observed in measurements of the $\Gamma_{15}$ component. ${ }^{11}$ However, when higher orders are considered, the increasing number of phonons participating in a specific peak causes the associated direct product representations to contain $\Gamma_{1}$ and $\Gamma_{15}$ irreducible representations for almost any possible set of constituent modes, thus rendering polarization analysis irrelevant. Previous to the experiments reported here, we performed several trials using different polarizations which confirmed that the relative intensities between the observed peaks of a specific order remain unaltered for orders higher than two. Therefore, in order to maximize the signal, which is specially critical for high orders, the spectra presented in this paper were recorded without polarization analysis.

In the second-order spectrum we focused only in the region between 600 and $700 \mathrm{~cm}^{-1}$, where combinations and overtones of the optical branches occur. ${ }^{10}$ In Table I we list the frequencies of the different peaks, their respective assignations, the comparison with their expected position, and their relative intensities and widths. The intensities were normalized to the highest intensity peak displayed in the paper, which corresponds to the wellknown two LO phonon peak. The intensity of this peak was found to be 17.8 times lower than that of the one LO

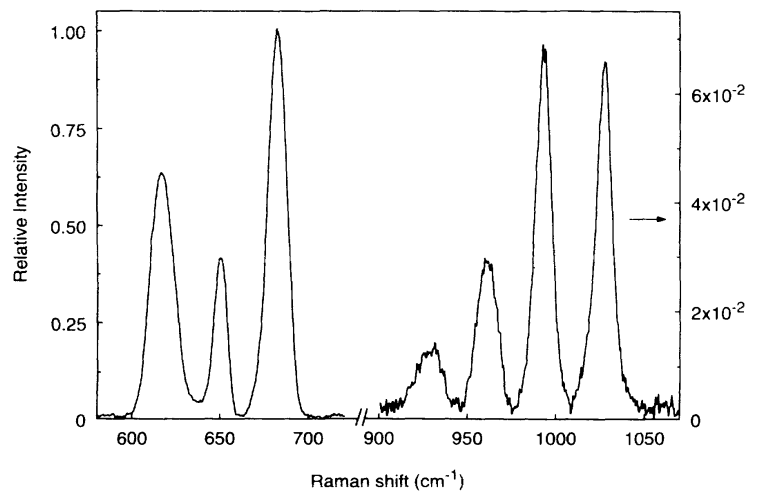

FIG. 1. Second- and third-order unpolarized Raman spectra of InP at room temperature. The intensity scale has been normalized to the intensity of the $2 \mathrm{LO}$ peak. The small residual background has been subtracted for clarity.
TABLE I. Frequencies of the peaks measured in the higherorder Raman spectrum of InP. We also give their assignation, a comparison with their expected position, and their relative intensities and respective linewidths. The intensities have been normalized to the $2 \mathrm{LO}$ peak and for the fourth and fifth orders they have been measured with respect to the average background noise level. The relative deviation is defined as $[\omega-\omega(n \Gamma)] / \omega(n \Gamma)$, where $\omega(n \Gamma)$ is the expected frequency obtained from the values $\omega_{\mathrm{TO}}(\Gamma)=306.9 \mathrm{~cm}^{-1}$ and $\omega_{\mathrm{LO}}(\Gamma)=344.6 \mathrm{~cm}^{-1}$ measured in this work.

\begin{tabular}{ccccc}
\hline $\begin{array}{c}\text { Raman } \\
\text { shift } \\
\omega\left(\mathrm{cm}^{-1}\right)\end{array}$ & Assignation & $\begin{array}{c}\text { Relative } \\
\text { deviation }\end{array}$ & Intensity & $\begin{array}{c}\text { Linewidth } \\
\left(\mathrm{cm}^{-1}\right)\end{array}$ \\
\hline 617 & 2 TO & $+5.2 \times 10^{-3}$ & $6.2 \times 10^{-1}$ & 16 \\
650 & TO+LO & $-1.7 \times 10^{-3}$ & $4.0 \times 10^{-1}$ & 9 \\
682 & 2LO & $-1.0 \times 10^{-2}$ & 1.00 & 12 \\
930 & 3TO & $+1.0 \times 10^{-2}$ & $1.1 \times 10^{-2}$ & 17 \\
962 & 2TO+LO & $+3.3 \times 10^{-3}$ & $2.7 \times 10^{-2}$ & 14 \\
994 & TO+2LO & $-2.4 \times 10^{-3}$ & $6.6 \times 10^{-2}$ & 11 \\
1028 & 3LO & $-5.8 \times 10^{-3}$ & $6.4 \times 10^{-2}$ & 11 \\
1274 & 3TO+LO & $+7.0 \times 10^{-3}$ & $3 \times 10^{-3}$ & 18 \\
1304 & 2TO+2LO & $+9 \times 10^{-4}$ & $6 \times 10^{-3}$ & 15 \\
1337 & TO+3LO & $-2.5 \times 10^{-3}$ & $1.4 \times 10^{-2}$ & 12 \\
1373 & 4LO & $-3.6 \times 10^{-3}$ & $1.9 \times 10^{-2}$ & 10 \\
1650 & 2TO+3LO & $+1.3 \times 10^{-3}$ & $2 \times 10^{-3}$ & 17 \\
1683 & TO+4LO & $-1.2 \times 10^{-3}$ & $5 \times 10^{-3}$ & 17 \\
1719 & 5LO & $-2 \times 10^{-4}$ & $5 \times 10^{-3}$ & 12 \\
\hline \hline
\end{tabular}

phonon peak. The peaks measured at 617,650 , and 682 $\mathrm{cm}^{-1}$ are in good agreement with the expected $2 \mathrm{TO}(\Gamma)$, $\mathrm{TO}(\Gamma)+\mathrm{LO}(\Gamma)$, and $2 \mathrm{LO}(\Gamma)$ phonons. However, we note from Table I that the 2TO and 2LO peaks have a considerable width and display a larger relative deviation from their expected positions than peaks of higher orders. Bearing in mind that neutron inelastic scattering measurements $^{7}$ indicate very flat longitudinal- and transverse-optical branches, it is probable that both peaks contain contributions of phonons from different points of the Brillouin zone. According to phonon-dispersion measurements, ${ }^{7}$ the longitudinal branch at point $L$ is located only $4.5 \mathrm{~cm}^{-1}$ below the frequency we found at point $\Gamma$, and hence contributions from both points presumably occur in the measured 2LO peak. On the contrary, considering that $2 \mathrm{TO}(L)$ lies $17 \mathrm{~cm}^{-1}$ above the measured peak, it seems unlikely that the 2TO peak has contributions from the point $L$ as it was previously suggested. ${ }^{10}$ In this case, in agreement with Alfrey and Borcherds, ${ }^{8}$ we think that the peak is mainly due to $2 \mathrm{TO}$ phonons at point $\Gamma$, where the minimum of the transverse branch over the whole Brillouin zone occurs. Nevertheless, the large width of this peak and its relative deviation towards high frequencies (see Table I) suggest additional contributions of TO phonons from other points of the Brillouin zone. Similarly, the participation of $\mathbf{k} \neq \mathbf{0}$ phonons in the 2LO peak may explain the comparatively large width of that peak as well as its relative deviation.

The distinct triplet formed by the second-order overtones and combinations of optical phonons led us to investigate possible recurrences of these features at higher orders. In the region between 900 and $1100 \mathrm{~cm}^{-1}$ we 
found four peaks, at $930,962,994$, and $1028 \mathrm{~cm}^{-1}$. They correspond to third-order Raman scattering and can be assigned to $3 \mathrm{TO}, 2 \mathrm{TO}+\mathrm{LO}, \mathrm{TO}+2 \mathrm{LO}$, and $3 \mathrm{LO}$ phonons at zone center, respectively. The agreement with the corresponding multiples of the LO and TO modes is very good, as shown in the third column of Table $I$. The intensity of these third-order phonons is about eighteen times weaker than their second-order counterparts whereas their widths are similar. To the best of our knowledge, this constitutes the first experimental observation of participation of transverse modes in multiphonon Raman processes of order higher than two in a zincblende-type semiconductor.

Scanning higher-energy regions, another group of four peaks was detected between 1200 and $1400 \mathrm{~cm}^{-1}$, as can be seen in Fig. 2. These peaks, measured at 1274, 1304, 1337 , and $1373 \mathrm{~cm}^{-1}$, correspond to fourth-order Raman scattering and we assign them to $\mathrm{LO}+3 \mathrm{TO}, 2 \mathrm{LO}+2 \mathrm{TO}$, $3 \mathrm{LO}+\mathrm{TO}$, and $4 \mathrm{LO}$ phonons at zone center, respectively. As it can be seen in Table $I$, there is also a very good agreement between the position of the peaks and the corresponding multiples of the transverse and longitudinal optical phonon frequencies at zone center. The intensity of the fourth-order peaks is only about four times weaker than their third-order counterparts, in contrast with the drastic intensity reduction observed when passing from second to third order. In the third- and fourth-order spectra it can be observed that the width of the peaks increases with the number of transverse phonons participating in the peak. In particular, for all orders, the peaks with participation of at least two transverse phonons show a significant broadening when compared with the rest of the peaks. The broadening of those peaks presumably arises from the contribution of $\mathbf{k} \neq 0$ phonons from the transverse optical branch, in a similar fashion as discussed for the broadening of the second-order peaks.

The peak observed in Fig. 2 at $1555 \mathrm{~cm}^{-1}$ corresponds to the vibrational mode of molecular oxygen. ${ }^{12}$ By purging with argon, the lines associated with the coupling between vibrational and rotational modes of the $\mathrm{O}_{2}$ molecule were removed, but the vibrational line, although

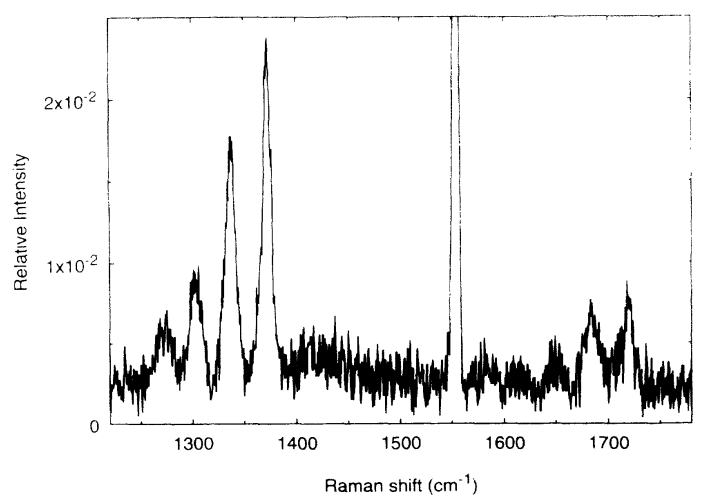

FIG. 2. Fourth- and fifth-order unpolarized Raman spectra of InP at room temperature. The intensity scale has been normalized to the intensity of the $2 \mathrm{LO}$ peak. A small residual background has been subtracted. The strong Raman line at $1555 \mathrm{~cm}^{-1}$ corresponds to the vibrational mode of atmospheric $\mathrm{O}_{2}$. strongly attenuated, was not completely suppressed by the flux we used. At higher energies it is also possible to clearly identify three small peaks, at 1650, 1683, and 1719 $\mathrm{cm}^{-1}$, as can be seen in Fig. 2. They correspond to fifthorder Raman scattering and can be assigned to $2 \mathrm{TO}+3 \mathrm{LO}, \mathrm{TO}+4 \mathrm{LO}$, and $5 \mathrm{LO}$ phonons. As shown in Table I, their frequencies are in excellent agreement with the corresponding multiples of the LO and TO modes at zone center. In all cases, and for all orders, the maximum difference between experimental frequencies and the corresponding multiples of the LO and TO frequencies is $1 \%$. The intensities of the fifth-order phonons are about one-third of those of fourth order. Thus, we differentiate two groups of intensity ratios. Considering, for instance, the $n$ LO peaks, we find that the ratio $I(n \mathrm{LO}) / I((n+1) \mathrm{LO})$ is approximately 18 for $n=1,2$ whereas for $n>2$ it turns out to be about 3 . These intensity ratios between consecutive orders do not agree with the theoretically predicted variation of the $n$th Raman cross section for overtones in semiconductors measured above the gap. ${ }^{13}$ The variation of the intensities with the order of the spectrum is also far from the $n^{-4}$ dependence proposed for $n \mathrm{LO}$ phonons under resonance in some II-VI compounds. ${ }^{14}$

A number of semiconductors with the zinc-blende structure present a transverse-optical branch with little dispersion over the whole Brillouin zone whereas a bigger dispersion is generally found along the longitudinaloptical branch. This is not the case for indium phosphide, for which, as it has already been mentioned, phonon dispersion studies ${ }^{7,15}$ indicate that the longitudinaloptical branch is almost as flat as the transverse-optical branch over the entire Brillouin zone. Calculations of two-phonon density of states using the overlap valence shell model ${ }^{15}$ show two very sharp and well-separated peaks corresponding to $2 \mathrm{LO}$ and $2 \mathrm{TO}$ phonons. We believe that the exceptional flatness of both optical branches, which gives rise to a very high density of states around $\operatorname{TO}(\Gamma)$ and $\mathrm{LO}(\Gamma)$, constitutes a key factor in making it possible to observe multiphonon lines up to fifth order.

\section{CONCLUSIONS}

It has been proved that it is possible to observe multiphonon lines in nonresonant Raman scattering in semiconductors. Up to five Raman-scattering orders could be detected in InP using incident light above the band gap but far from any interband transition of this compound. These experiments allowed us to observe not only longitudinal phonons but also transverse ones. All the observed multiphonon lines show a very good agreement with phonons of the form $p \mathrm{LO}(\Gamma)+q \mathrm{TO}(\Gamma)$, with $p+q=n$ the order of the spectrum. We expect that the characteristics of the higher-order phonons reported in this work will yield clues for a better understanding of the mechanisms involved in multiphonon Raman scattering.

\section{ACKNOWLEDGMENT}

We gratefully acknowledge the Spanish Ministerio de Educación y Ciencia for financial support. 
${ }^{1}$ R. C. C. Leite, J. F. Scott, and T. C. Damen, Phys. Rev. Lett. 22, 780 (1969).

2J. F. Scott, R. C. C. Leite, and T. C. Damen, Phys. Rev. 188, 1285 (1969).

${ }^{3}$ J. F. Scott, T. C. Damen, W. T. Silfvast, R. C. C. Leite, and L. E. Cheesman, Opt. Commun. 1, 397 (1970).

${ }^{4}$ S. S. Islam, Shyama Rath, K. P. Jain, S. C. Abbi, C. Julien, and M. Balkanski, Phys. Rev. B 46, 4982 (1992).

${ }^{5}$ T. Ruf and M. Cardona, Phys. Rev. Lett. 63, 2288 (1989).

${ }^{6} \mathrm{R}$. T. Phillips, T. Ruf, and M. Cardona, in 20th International Conference on the Physics of Semiconductors, Thessaloniki, 1990, edited by E. M. Anastassakis and J. D. Joannopoulos (World Scientific, Singapore, 1990), Vol. 3, p. 1993.

${ }^{7}$ P. H. Borcherds, G. F. Alfrey, D. H. Saunderson, and A. D. B. Woods, J. Phys. C 8, 2022 (1975).

${ }^{8}$ G. F. Alfrey and P. H. Borcherds, J. Phys. C 5, L275 (1972).

${ }^{9}$ E. Bedel, G. Landa, R. Carles, J. P. Redoulès, and J. B. Renuc- ci, J. Phys. C 19, 1471 (1986).

${ }^{10}$ R. Trommer, H. Müller, M. Cardona, and P. Vogl, Phys. Rev. B 21, 4869 (1980).

${ }^{11}$ M. Cardona, in Light Scattering in Solids II, edited by M. Cardona and G. Güntherodt, Topics in Applied Physics Vol. 50 (Springer-Verlag, Berlin, 1982); also, see W. Kiefer, W. Richter, and M. Cardona, Phys. Rev. B 12, 2346 (1975); R. Carles, N. Saint-Cricq, J. B. Renucci, M. A. Renucci, and A. Zwick, ibid. 22, 4804 (1980); R. L. Schmidt, K. Kunc, M. Cardona, and H. Bilz, ibid. 20, 3345 (1979).

${ }^{12}$ See, e.g., Raman Spectroscopy of Gases and Liquids, edited by A. Weber, Topics in Current Physics Vol. 11 (Springer, Berlin, 1979).

${ }^{13}$ R. Zeyher, Solid State Commun. 16, 49 (1975).

${ }^{14}$ Z. C. Feng, S. Perkowitz, and P. Becla, Solid State Commun. 78, 1011 (1991).

15P. H. Borcherds and K. Kunc, J. Phys. C 11, 4145 (1978). 